\title{
À la recherche du figement perdu : le figement cognitif
}

\author{
Fasciolo, Marco, Meneses-Lerin, Luis, \& Zhu, Lichao \\ Fasciolo, Marco \\ LDI (Lexiques, Dictionnaires, Informatique) UMR 7187 \\ Université Paris 13 \\ marco.fasciolo@gmail.com \\ Meneses-Lerin, Luis \\ LDI (Lexiques, Dictionnaires, Informatique) UMR 7187 \\ Université Paris 13 \\ luisonlerin@yahoo.com \\ Zhu, Lichao \\ LDI (Lexiques, Dictionnaires, Informatique) UMR 7187 \\ Université Paris 13 \\ lichao.zhu@hotmail.com
}

\section{Introduction}

Le figement perdu serait le figement cognitifi. Le phénomène du figement (Gross 1996, Mejri 1997) a été systématiquement abordé sous un angle purement linguistique et formel. Linguistiquement, ce sont les signifiants d'une séquence qui se figent (par exemple, une séquence prédicative comme prendre le taureau par les cornes) pour aboutir à un seul signifiant complexe qui code un nouveau signifié simple, non compositionnel et idiomatique. Nous proposons d'aborder le phénomène du figement directement sous un angle cognitif. Dans cette perspective, ce sont des liens d'implication entre des idées qui se figent (par exemple, entre «Écossais» et «radin») pour aboutir à des inférences «toutes faites» et idiomatiques (Il est Écossais = Il est radin).

Certes, la pertinence cognitive du phénomène du figement, en soi, n'est pas une nouveauté : elle a toujours été reconnue dans l'importance accordée à la notion de stéréotypie. Cependant, elle n'a jamais fait l'objet d'une formulation claire. Dans cette perspective, nous cherchons à formuler explicitement la thèse selon laquelle le figement n'est pas un phénomène unique mais double : il existe un processus de figement linguistique et un processus de figement cognitif qui fonctionnent en parallèle. En conséquence, le réseau de notions pertinentes du premier processus (par exemple : combinatoire vs. contrainte, degré de contrainte, collocation, appropriation...) trouvent des analogies au niveau cognitif du deuxième processus.

Une formulation explicite de la thèse du double volet - linguistique et cognitif - du figement permet, entre autres, de poser d'une façon claire la problématique des formes de leur interaction (cf. par les structures être Adj comme SN, Mejri 1994 ${ }^{\mathrm{ii}}$, Anscombre 2011).

Nous n'avons pas la prétention d'aboutir à des résultats exhaustifs; notre but est de montrer le bien fondé de la distinction - entre figement linguistique et figement cognitif - qui est à la base du traitement des séquences figées. Nous poursuivrons en trois étapes :

- en montrant que les propriétés définitoires du figement linguistique ont des analogies cognitives,

- en montrant que distinguer figement linguistique du figement cognitif permet de stratifier la notion de stéréotype,

- en montrant, pour boucler la boucle, qu'il y a aussi un défigement cognitif comme il y a un défigement linguistique. 


\section{Figement linguistique et figement cognitif}

Dans des séquences verbales telles que prendre le taureau par les cornes, les carottes sont cuites ou casser sa pipe, le figement peut être abordé sous deux angles : lexical, concernant le rapport de nonmotivation entre un signifiant (complexe) et son signifié idiomatique et syntaxique, concernant le blocage des transformations.

\subsection{Non-motivation synchronique et motivation diachronique}

Il est connu qu'une expression figée est synchroniquement non-motivée. Cette non-motivation synchronique est le pendant d'une motivation diachronique (à ce propos, cf. par exemple le $§ 10$. de Gross [1996: 21-22]). En d'autres termes, étant donné qu'une expression figée n'est pas motivée, il faut chercher les raisons historiques qui ont amené cette séquence à fonctionner comme signifiant complexe d'un signifié non-compositionnel. Voici un exemple :

(1) a. Il a cassé l'ordinateur.

b. Il a cassé sa pipe.

Dans (1), il est pertinent de se demander pour quelle(s) raison(s) la motivation diachronique se manifeste uniquement dans (b) mais pas dans (a). Dans l'énoncé (1b), la motivation peut nous sembler transparente ou opaque (si elle est perdue au fils du temps) tandis que dans l'énoncé (1a), le fait de parler de motivation diachronique, de transparence ou d'opacité, n'a aucun sens.

La notion de motivation que nous venons d'illustrer s'applique aussi bien aux idées qu'aux inférences. Comparons les exemples suivants :

(2) a. Il a neigé toute la nuit, donc les rues seront glissantes.

b. Il est Écossais, donc il est radin.

Dans les deux cas, donc fonctionne comme un « déclencheur » d'inférences : dans (2a), cette inférence conclut que « les rues seront glissantes » du fait qu'il a neigé toute la nuit; dans ( $2 \mathrm{~b})$, elle conclut que quelqu'un est « radin » parce qu'il est Écossais. Il est clair que s'interroger sur la motivation historique qui se trouve derrière la première inférence serait absurde, alors que s'interroger sur la motivation historique de la deuxième (et ne pas réussir à la reconstruire) serait tout à fait sensé. Tandis que l'inférence exprimée dans (2a) repose sur un lien causal qui fait l'objet d'un modèle cognitif partagé, vivant ; celle de l'inférence exprimée dans $(2 b)$ repose sur un cliché cristallisé dans l'histoire.

Si cela est vrai, alors l'inférence dans (2b) serait motivée historiquement au même titre que l'expression figée dans (1b)

\subsection{Sclérose argumentative}

Ce qui caractérise une expression en tant que figée ou qui rend compte de son degré de figement est sans doute sa «sclérose syntaxique» (Anscombre, 2011). Cette sclérose syntaxique qui se manifeste linguistiquement trouve son analogie au niveau cognitif avec le blocage argumentatif. Pour illustrer la notion de «blocage argumentatif », nous présenterons deux idées.

D'abord, songeons aux questions suivantes :

(3) a. Pourquoi s'il neige, les rues deviennent-elles glissantes ?

b. Pourquoi la neige rend-elle les rues glissantes ?

Dans (3), les deux questions portent sur le lien causal qui se trouve à l'origine de l'inférence dans (2a) : il a neigé toute la nuit, donc les rues sont glissantes. Il est évident que si l'on pose les questions sous (3) à quelqu'un qui affirme (2a), il risque d'être surpris car nous lui demandons de nous fournir une explication d'un modèle cognitif que tout le monde est censé partager. 
Abordons maintenant les questions dans (4):

(4) a. Pourquoi, si quelqu'un est Écossais, serait-il radin?

b. Pourquoi les Écossais seraient-ils radins?

Poser les questions (4) à quelqu'un qui affirme (2b) est la meilleure façon de mettre en évidence son préjugé sur les Écossais, parce que nous poussons la personne à prouver l'existence du lien causal entre « être Ecossais » et "être radin ». En d'autres termes, l'idée qui se trouve à la base de (2b) connaît un blocage de l'argumentation, tout comme l'expression figée Il a cassé sa pipe connaît un blocage au niveau des transformations syntaxiques.

\subsection{L'analogie: syntaxe et argumentation}

Les raisons qui nous amènent à qualifier de figées certaines expressions, nous conduisent également à qualifier de figées certaines idées toutes faites qui se manifestent au moyen des inférences. Si cela est vrai, le phénomène du figement connaît alors deux volets : le volet linguistique des expressions figées et le volet cognitif des idées ou inférences figées.

Nous émettons également une hypothèse sur la hiérarchie du figement, notamment le cas de « Adj comme $\mathrm{SN} »$ qui fait l'objet de nombreuses études. La plupart de linguistes considèrent que le syntagme « comme $\mathrm{SN} »$ est un intensifieur dans ce cas (Mejri : 2000, Anscombre : 2011, Leroy : 2007). Partant de ce consensus, nous pensons qu'il est évident de dire qu'il existe un niveau supérieur exprimant une fonction d'intenfication et des niveaux inférieurs dont le niveau cognitif actualisé : on déduit de "bête comme une oie » qu' « une oie, c'est bête » (Leroy : 2007) et non-actualisé : on ne peut pas déduire de « beau comme un camion » qu' « un camion, c'est beau » qui n'a aucun sens de nos jours, cela prouve que cette expression est figée dans la langue.

En outre, nous constatons que l'omission de l'adjectif serait permise dans certaines expressions «Adj comme SN ». Par exemple, de «Il est comme un âne », nous interprétons qu' " il est têtu », "il est bête » ou « il est bête et têtu » selon le contexte ${ }^{\mathrm{iii}}$. Quand l'idée que l'âne est bête ou têtu est ancrée dans la pensée du locuteur, il n'est alors plus question de langue. Nous remarquons que l'omission est notamment valide sur des expressions doxales et la même opération n'est pas envisageable sur des parangons comme « beau comme un camion », « bête comme ses pieds ».

\section{Les inférences figées}

A partir des conclusions tirées précédemment, nous considérons que le double volet - cognitive et linguistique - du figement est un fait bien fondé. En nous appuyant sur cette hypothèse, nous proposons de corroborer ultérieurement cette idée et de reposer la problématique concernant la stéréotypie.

\subsection{Le rapport entre figé et non-figé}

Commençons par une remarque qui, d'une certaine manière, boucle la boucle de l'argumentation à peine déroulée. Reprenons l'exemple (1b). L'interprétation d'une SF comme Il a cassé sa pipe est nécessairement double: d'un côté, il y a la lecture idiomatique ou non compositionnelle, de l'autre la lecture dite non-idiomatique ou compositionnelle. Or, on retrouve cette même duplicité au niveau cognitif comme les questions de (4).

Comme on l'a vu sous $\S 2.2$, les questions que l'on pose sous (4) représentent la meilleure façon de souligner le préjugé qui consiste à penser que tous les Écossais sont radins. Mais si le stéréotype est valide, les questions sous (4) nous obligent à réinterpréter cette idée-clichée ou cette « catachrèse » d'idée en tant qu'idée cognitivement vivante qui repose sur un lien causal censé être argumenté. Ce fait montre que, devant un énoncé qui exprime une idée cognitivement figée comme dans (2b), nous pouvons 
également envisager une lecture cognitivement non-figée exactement comme s'il s'agissait d'une expression.

Prenons également l'exemple suivant :

(5) Il est radin comme un Écossais.

L'énoncé (5) connaît deux interprétations, toutes deux compositionnelles. D'une part, le déterminant un reçoit une lecture générique, qui concerne les propriétés liées au concept d'Écossais et qui exprime le cliché qui se trouve à la base de l'inférence (2b); d'autre part, le déterminant un reçoit une lecture spécifique (un Écossais que j'ai connu il y a longtemps dans un pub d'Édimbourg) qui met en jeu les propriétés du référent contingent du mot Écossais qui n'a aucun lien avec ce cliché. Dans ce cas, le déterminant un a plutôt une «valeur spécifique » (Leroy, 2007). Ainsi, nous constatons le passage d'une lecture cognitivement figée à une lecture cognitivement non-figée.

\subsection{On dit que... vs. On dit « ... »}

Une propriété très connue, mais controversée, est celle qui permet aux expressions de se combiner avec des verbum dicendi au sujet indéfini. Comparons par exemple :

(6) a. On dit: les carottes sont cuites.

b. ? On dit: aujourd'hui je suis allé au théâtre.

Cette propriété nous permet de souligner ultérieurement - c'est-à-dire de faire une analogie qui se déroule à deux niveaux différents - le figement cognitif et le figement linguistique. Afin d'illustrer ce que nous venons de dire, comparons les exemples (7) et (8) :

(7) a. On dit que les Écossais sont radins.

b. Pourquoi dit-on que les Écossais sont radins?

(8) a. ? On dit que la neige rend les rues glissantes.

b. ? Pourquoi dit-on que la neige rend les rues glissantes ?

Les énoncés (7a) et (8a) expriment les idées qui se trouvent à la base ${ }^{\text {iv }}$ des inférences dans (2b) et dans (2a) ; tandis que les énoncés sous $(7 b)$ et $(8 b)$ questionnent les causes des inférences. Cela prouve que, contrairement à (7), la subordonnée complétive de (8) exprime un lien causal objectif qui ne dépend d'aucune vox populi. Une idée figée, comme dans les Écossais sont des radins, peut fonctionner comme une complétive d'un verbum dicendi au sujet indéfini, ce qui diffère d'une connaissance encyclopédique telle que la pluie peut faire déborder les fleuves. Cela rapproche cette idée figée des expressions figées (du type de [6a]). Cependant - le lecteur l'aura immédiatement remarqué - dans (7) on emploie l'expression On dit que qui concerne le contenu propositionnel de la subordonnée, alors que dans l'expression figée sous (6a) on trouve simplement la forme On dit «... ». Dans (6a), ce qui suit le verbum dicendi n'est pas envisagé par son contenu conceptuel mais plutôt en tant qu'expression linguistique (ou en suppositio materialis ou bien en modalité « autonymique »). C'est la différence entre les deux niveaux - conceptuel et formel - de la précédente analogie.

\subsubsection{La symétrie du figement}

Les remarques faites sous $\S 2.1$. nous offrent non seulement une deuxième possibilité de confirmer les idées présentées dans $\S 1$, mais elles nous permettent également d'envisager la nécessité de décomposer la notion de stéréotype. Pour illustrer ce que nous entendons par là, comparons les exemples (5) et (9a) :

(9) a. Cela est noir comme l'encre / l'ébène / le charbon..

Les comparaisons dans (5) et (9a) reposent sur des idées qu'on pourrait synthétiser comme les Écossais sont radins et l'encre (l'ébène, etc.) est noire et que l'on pourrait appeler des stéréotypes. Le problème 
consiste à mettre en évidence la différence qui existe entre ces deux énoncés. Grâce à la structure on dit que, cette différence devient évidente:

(9) b. ? On dit que le charbon est noir.

On peut bien affirmer avec cohérence (7a), mais pas (9b) tout comme on ne peut pas affirmer (8a). La différence dans le comportement de la structure on dit découle du fait que le charbon soit «noir» est objective, ancrée dans des références naturelles et stables, et que l'on présuppose que tout le monde la partage. En revanche, l'idée que les Écossais sont « radins » est le résultat d'une évaluation sociale que l'on est censé reconnaître seulement à l'intérieur d'une communauté d'une langue donnée. C'est pourquoi nous proposons d'appeler idée figée l'exemple présenté dans (7a), ce qui n'est pas le cas pour les exemples sous $(8 \mathrm{a})$ et $(9 \mathrm{~b})$.

À ce propos, il est important de souligner que nous n'avons pas la prétention de classer les niveaux de partage de ces idées. Cependant nous voulons pointer les différences présentées au niveau épistémique et, chemin faisant, mettre l'accent sur la nécessité de distinguer des «niveaux » à l'intérieur de la catégorie des stéréotypes. La notion d'idée figée serait justement, à notre avis, l'un des niveaux de cette catégorie.

\subsubsection{Pour une analyse de la notion de stéréotypie}

Analysons les exemples suivants :

(10) a. Il est Ecossais : c'est-à-dire, il est radin.

b.? Il est Allemand : c'est-à-dire, il est grand et blond.

Dans (10a), l'emploi de c'est-à-dire est naturel et analytique, ce qui n'est pas le cas dans (10b) qui est synthétique ou explicatif. Cela nous permet de distinguer le "stéréotype" les Écossais sont des radins du "stéréotype" les Allemands sont grands et blonds. Certes, ce dernier est sans doute aussi un stéréotype ; cependant - comme la différence entre (10a) et (10b) le montre - il n'est pas cognitivement figé, mais vivant. En effet, radin peut être un synonyme d'Écossais car il y a une inférence figée qui crée un emploi idiomatique du mot Écossais, ce qui n'est pas le cas pour Allemand car, ici, il n'y a aucune inférence figée.

Il est également important de souligner que la distinction entre idées figées et expressions figées permet de comprendre d'une façon très naturelle les remarques d'Anscombre (2011: 27-35) concernant les parangons de la forme être Adj. comme $S N$. À notre avis, il existe, d'une part, le moule formel être Adj. comme $S N$ et d'autre part, le matériel cognitif avec lequel on remplit ce moule. Si ce matériel cognitif est figé, comme dans les Écossais sont radins ou les Polonais sont de grands buveurs, le résultat en est un parangon figé : par exemple, être radin comme un Écossais, être soûl comme un Polonais. Mais si le matériel cognitif n'est pas figé comme dans les Allemands sont blonds et grands, le résultat en est un parangon cognitivement vivant ${ }^{\mathrm{V}}$.

De plus, la distinction entre figement cognitif et figement linguistique nous permet de poser la question sur leur rapport. Pour illustrer ce point, nous concevons le schéma suivant :

$$
\text { Niveau formel : Manger les pissenlits par la racine } \quad v s . \quad \begin{gathered}
\text { Être radin comme un Écossais } \\
\downarrow
\end{gathered}
$$

Niveau cognitif : Les personnes meurent et elles sont enterrées. vs. Les Écossais sont radins.

Les niveaux cognitif et linguistique qu'on vient de représenter sont symétriques. Le niveau cognitif présente des notions partagées : des notions vivantes telles que les gens meurent et qu'on les enterre ; et des notions figées telles que les Écossais sont radins. Inversement, le niveau formel présente la structure figée : la locution manger les pissenlits par la racine et la structure non figée : être Adj. comme SN.

Or, les flèches indiquent que cette symétrie implique deux directions d'action opposées d'un niveau à l'autre. En ce qui concerne l'expression manger les pissenlits par la racine, le figement linguistique fixe, dans une seule locution, deux concepts virtuellement indépendants (certains verront ici un procès de 
«dénomination» [Mejri, 2000]). En revanche, en ce qui concerne être radin comme un Écossais, le figement cognitif cristallise une structure syntaxiquement libre.

\subsection{Les deux volets du figement}

Les résultats obtenus jusqu'ici peuvent être synthétisés de la façon suivante. Au $\S 2$, nous avons défendu l'hypothèse qu'il y a des idées qui sont figées au même titre que les expressions figées. Ces deux aspects idées figées et expressions figées - constituent deux volets d'un même phénomène, le figement : le volet cognitif et le volet formel. Au $\S 3$, nous avons présenté deux distinctions: nous avons distingué, à l'intérieur des connaissances partagées, les idées figées des connaissances encyclopédiques cognitivement vivantes; nous avons posé d'une façon explicite la question du rapport entre le figement cognitif et le figement linguistique.

Dans ce qui suit, nous nous intéresserons à la question suivante : existe-t-il une contrepartie cognitive du défigement linguistique (Gross 1996 : 19-20) ?

\section{Y a-t-il le défigement cognitif ?}

La question soulevée sous $\S 3.3$. nous paraît cruciale car le défigement est considéré l'un des meilleures indices du figement (Mejri, Lecler). En effet, puisque le défigement présuppose le figement, le fait qu'une séquence puisse être défigée implique qu'elle était figée. Si nous pouvons déterminer une contrepartie cognitive du défigement linguistique - défigement cognitif - nous aurions par là trouvé un autre argument pour le double volet cognitif et linguistique du phénomène du figement.

Nous commençons donc par proposer une analyse plausible du défigement linguistique et par là, nous envisageons une définition du défigement cognitif.

\subsection{Du défigement linguistique au défigement cognitif}

Le défigement « consiste à ouvrir des paradigmes là où, par définition, il n'y en a pas » (Gross, $1996: 20$ ). C'est-à-dire, «Toute atteinte à la fixité formelle et à la globalité sémantique des SF serait considérée comme un défigement » (Mejri, 2009 : 158) Voici un exemple :

(11) a. La vie en rose.

A partir du titre de la chason d'Edith Piaf, nous pouvons envisager un jeu de mots comme :

(11) b. La ville en rose.

Laissons de côté la question s'il faut ou non considérer un titre de chanson comme une SF. Ce qui nous concerne est que, selon les critères de Gross (1996:19-20), le jeu susmentionné serait un exemple de défigement. Mais qu'est-ce que le défigement en concret?

Nous proposons de l'analyser en distinguant deux composantes: des conditions requises et un déclenchement du 'jeu'.

(i) En amont - au niveau des conditions requises - il y acte de référence grâce auquel l'énoncé (11b) évoque l'expression (11a). Et la reconnaissance du défigement est toujours directement liée à la SF (Mejri, Lecler).

(ii) En aval - au niveau d'activation du jeu de mots - il y a un contraste entre (11b) et (11a), qui s'appuie justement sur l'expression figée (11a) et qui ouvre une palette d'interprétations a priori imprévisibles.

Le point (ii) peut être envisagé comme une implicature conversationnelle griceenne (Gréciano, 1986) qui exploite la SF (11a). Ce qui est tout à fait cohérent avec l'idée que le défigement soit «le meilleur révélateur du figement». En synthèse, notre analyse du défigement linguistique présentée met en évidence trois points : 
a) la référence a une entité ;

b) cette entité est une SF ;

c) cette SF fonctionne comme pivot d'une implicature conversationnelle.

C'est à partir de cette analyse que nous allons définir la notion de défigement cognitif. Le défigement cognitif suit le même processus. Toutefois, la nature du pivot de l'implicature conversationnelle n'est pas linguistique mais cognitive, soit :

a) la référence a une entité ;

b) cette entité est une idée figée ;

c) cette idée figée fonctionne comme pivot d'une implicature conversationnelle.

Le défigement cognitif s'attaque justement à des idées figées avant de s'opérer sur la langue. Le processus cognitif est donc mis en avant pour diriger le défigement. Nous allons maintenant proposer deux exemples que nous considérons particulièrement représentatifs.

\subsection{Exemples sur le défigement cognitif}

\subsubsection{Premier exemple}

Le premier exemple est des énoncés suivants :
a. Il est généreux comme un Ecossais.
b. Il est sobre comme un Polonais.

Dans (12), nous apercevons d'emblée une ironie. Cette ironie découle d'un contraste entre le contenu de l'adjectif sobre, généreux et l'idée figée que les Polonais sont des ivrognes et que les Ecossais sont radins $^{\mathrm{vi}}$. Comme (11b), nous pouvons analyser (12) avec la notion d'implicature conversationnelle et distinguer les composants suivants :

(i) un acte de référence. Les exemples de (12) évoquent les idées figées ;

(ii) l'interprétation contrastive entre le contenu sémantique de (12) et ces idées figées

Contrairement au (11b), les exemples de (12) ne s'appuient pas sur la langue, mais sur les idées figées. Nous pouvons donc affirmer l'exitence du défigement cognitif.

\subsubsection{Deuxième exemple}

Le 11 Octobre 2008, Jörg Haider, homme politique autrichien de l'extrême droite, meurt à cause d'un accident de voiture. Quatre jours après, sur Le Canard Enchainé on trouve :

(13) Jörg Haider se tue en voiture : Au lieu de lever le bras... il aurait dî lever le pied.

Ce qui nous intéresse est le rapport entre lever le bras et lever le pied. La deuxième est une SF qui signifie « cesser d'appuyer sur l'accélérateur »; la première, en revanche, n'a rien de figé.

La présence de Jörg Haider dans le cotexte immédiat et son appartenance politique permettent d'interpréter l'expression lever le bras par rapport à l'idée figée qu'un politicien d'extrême droite est censé employer le salut nazi. Cette interprétation de lever le bras réagit avec la SF lever le pied en la défigeant. Ce cas de figure est différent de l'exemple précédent, parce que le défigement linguistique a lieu grâce à une idée figée. Il nous montre l'influence d'une idée figée sur une SF. 


\section{Conclusion : la valeur heuristique du figement cognitif}

Les deux exemples de défigement cognitif que nos venons de montrer nous indiquent des pistes de recherche.

Le premier exemple montre que le défigement cognitif doit être étudié sur le terrain des effets ironiques. De plus, il nous induit à envisager une typologie des idées figées selon les effets (comique, ironique, cynique, etc.) déclenchés par leur défigement. Il est raisonnable que chacun de ses effets soit liée au type de l'idée figée sur lequel il s'appuie. Le deuxième, en revanche, nous induit à examiner l'interaction entre idées figées et expressions figées, c'est-à-dire entre les deux volets du figement - cognitif et linguistique qui ont fait l'objet de cet article.

\section{Références bibliographiques}

ANSCOMBRE, J.C. (2001). Le rôle du lexique dans stéréotypes dans la théorie des stéréotypes. Langages, 142, 5576.

ANSCOMBRE, J.C. (2011). Figement, idiomaticité et matrices lexicales. Le figement linguistique : la parole entravée, $17-40$.

GRECIANO, G. (1986). Les inférences de l'idiome. Travaux de Linguistique et de Littérature, 24 (1), 139-153.

GRICE, H. P. (1975). Logic and conversation. Syntax and Semantics 3 - Speech Acts, 41-58.

GROSS, G. (1996). Les expressions figées en français: noms composés et autres locutions. Paris : Ophrys.

LECLER, A. (2007). Le défigement : un nouvel indicateur des marques du figement ?. Cahiers de praxématique, 46, 43-60.

LEROY, S. (2007). Les comparaisons « comme SN » exprimant le plus haut degré. Travaux de linguistique, 54, 6982.

MEJRI, S. (1994). Séquences figées et expression de l'intensité : essai de description sémantique. Cahiers de Lexicologie, 65, 111-122.

MEJRI, S. (2000). Figement et dénomination. Meta : journal des traducteurs 45, 4, 609-621.

MEJRI, S. (2009). Figement, défigement et traduction : problématique théorique. Figement, défigement et traduction, Encuentros Mediterráneos vol. 2, 153-163.

SCHAPIRA, C. (2000). Les stéréotypes du français : proverbes et autres formules. Paris : Ophrys.

\footnotetext{
${ }^{1}$ Comme Schapira oppose « stéréotypes de pensée » à « stéréotypes de langue » (1999), le figement cognitif que nous désignons englobe les stéréotypes, les clichés, la doxa, les préjugés dans le sens où ce dernier s'oppose au figement linguistique (formel). Le figement cognitif est l'ensemble des idées figées, qui n'a pas besoin d'intervention langagière, dans une langue donnée.

${ }^{2}$ S. Mejri étudie dans son article le mécanisme de séquence figée (SF) et son rapport avec l'intensité. Notre article fait echo à certains de ses arguments.

${ }^{3}$ La relation entre les stéréotypes et le référent n'est d'ailleurs pas toujours univoque. Un référent peut correspondre à plusieurs stéréotypes, et vice versa.

${ }^{4} \mathrm{Ou}$ bien qui fonctionnent comme prémisses.

${ }^{5}$ On peut s'attendre à ce que les langues se différencient en ce qui concerne le matériel cognitif figé que l'on met dans des moules (par exemple, être Adj. comme $S N$ ) qui restent constants. Voilà une autre analogie entre figement cognitif et linguistique.
} 
(C) aux auteurs, publié par EDP Sciences, 2012

${ }^{6}$ Concernant les déterminants indéfinis dans une séquence figée de ce genre, Leroy pense qu'ils «effectuent clairement une référence générique » (2007: 73) 